\title{
MAGNETIC FIELDS IN STAR-FORMING REGIONS OF OUR GALAXY
}

\author{
B. HUTAWARAKORN AND R. J. COHEN
}

NRAL, University of Manchester, Jodrell Bank, Macclesfield, Cheshire, SK11 9DL, UK

Masers provide a direct way of measuring magnetic fields in star-forming regions. $\mathrm{OH}$ ground-state masers at $18 \mathrm{~cm}$ wavelength exhibit strong circular polarization due to Zeeman splitting. The implied magnetic field strength is typically a few $\mathrm{mG}$, which is sufficient for the field to be dynamically important, e.g. in channelling the observed bipolar outflows. Moreover there are indications that magnetic fields in maser regions are aligned with the large-scale Galactic magnetic field (Reid \& Silverstein 1990), and that bipolar molecular outflows are also aligned with the local Galactic magnetic field (Cohen, Rowland \& Blair 1984). Some theoretical work in fact suggests that the magnetic field is intimately connected with the origin of the molecular outflow (e.g. Pudritz \& Norman 1983; Uchida \& Shibata 1985). It is therefore important to investigate the magnetic field configuration in these regions in as much detail as possible.

We report full-polarization spectral-line observations of $\lambda 18 \mathrm{~cm} \mathrm{OH}$ masers in three star-forming regions using MERLIN: G35.2-0.74N, W75N and NGC 7538. These three star-forming regions are clear examples of molecular disk and bipolar outflow structure, with the $\mathrm{OH}$ masers embedded at the centre of these regions. The $\mathrm{OH}$ masers lie typically within $\sim 1000 \mathrm{AU}$ of the central source. The distributions of the $\mathrm{OH}$ masers show the disk-like structure to be orthogonal to the bipolar outflow direction. We found strong linearly polarized masers (with a percentage of linear polarization more than 88\%) in W75N and NGC 7538-IRS 11.

In each source, the masers indicate an ordered magnetic field, which in some regions is aligned with the bipolar outflow while in others it is orthogonal to the direction of the outflow. The field strengths measured range from 2 to $8 \mathrm{mG}$, and are thus apparently relevant for the source evolution and dynamic. In G35.2-0.74N, W75N and NGC 7538-IRS 11, the 
field direction appears to reverse from one side of the disk to the other, suggesting a toroidal component of the magnetic field.

Furthermore, we discovered new 1665 and $1720 \mathrm{MHz} \mathrm{OH}$ masers in the region of NGC 7538-IRS 9. We also found new $1667 \mathrm{MHz} \mathrm{OH}$ masers in the region of IRS 11 , in addition to the $1665 \mathrm{MHz}$ masers previously mapped by Brebner (1988).

We compare our results with magnetic field configurations derived from other polarimetric measurements (e.g. Aitken et al. 1993; Vallee \& Bastien 1995). Our results appear to be in good agreement with the magnetic field configurations derived from those measurements, suggesting that the largescale ( $\sim 20$ arcsec) magnetic field is twisted in the disk region where the masers are embedded. This is the configuration predicted by Uchida \& Shibata (1985) and supports their hydromagnetic model against the alternative model proposed by Pudritz and Norman (1983).

\section{References}

Aitken D. K., Wright C. M., Smith C. H., Roche P. F., 1993, Mon. Not. R. Astr. Soc., 262,456

Brebner G. C., 1988, PhD thesis, University of Manchester

Cohen R. J., Rowland P. R., Blair M. M., 1984, Mon. Not. R. Astr. Soc., 210, 425

Pudritz R. E., Norman, C. A., 1983, Astrophys. J., 274, 677

Reid M. J., Silverstein E. M., 1990, Astrophys. J., 361, 483

Uchida Y., Shibata K., 1985, Publ. Astron. Soc. Japan, 37, 515

Vallee J. P., Bastien P., 1995, Astron. Astrophys., 294, 831 